Die Bundesbeauftragte für den Datenschutz und die Informationsfreiheit

\title{
Dialogkonferenz „Datenschutz für Kinder": Belange der Kinder stärker berücksichtigen
}

Die Bundesbeauftragte für den Datenschutz und die Informationsfreiheit (BfDI), Andrea Voßhoff, hat am 3. Juli 2018 zusammen mit dem Verein Deutschland sicher im Netz e. V. (DsiN) und dessen Projekt DigiBitS (Digitale Bildung trifft Schule), dem Berufsverband der Datenschutzbeauftragten Deutschlands e.V. (BvD) mit seiner Initiative „Datenschutz geht zur Schule“ sowie dem Institut für Medienforschung und Medienpädagogik der Technischen Hochschule Köln eine Konferenz ausgerichtet, auf der sich rund 200 Teilnehmer darüber ausgetauscht haben, welche Rolle der Datenschutz für Kinder im Umgang mit digitalen Medien spielt und an welchen Punkten noch Nachbesserungsbedarf besteht.

„Einmal unmittelbar von jungen Menschen zu erfahren, wie sie über das Thema Datenschutz im Zusammenhang mit den täglich von ihnen genutzten Medienangeboten denken und was aus ihrer Sicht wichtig ist, ist mir ein besonderes Anliegen. "Auf diese Weise umschrieb Andrea Voßhoff den Anstoß und die Motivation zur Ausrichtung einer Konferenz, die in dem dargebotenen Format für die BfDI ein Novum darstellt.

In ihrer Zusammenfassung der Ergebnisse der Konferenz resümierte Andrea Voßhoff:

„Bei der Vermittlung digitaler Kompetenzen in der Erziehung und im Unterricht sollte der Datenschutz für Kinder ein zentrales Thema sein. Dieses Thema sollte aber nicht alleine den Schulen auferlegt werden. Auch das Erziehungsumfeld der Kinder und Jugendlichen ist stärker einzubinden und aufzuklären, um Kindern auf die Risiken der neuen digitalen Welt vorzubereiten und für den Schutz eigener Daten zu sensibilisieren."

„Wir müssen uns als Gesellschaft bewusst werden, dass mit „Digitaler Bildung“ nicht allein die Ausstattung der Schulen gemeint sein darf, sondern gleichermaßen die Befähigung zum verantwortungsvollen Umgang mit eigenen Daten und Informationen über andere, “ ergänzt Rudi Kramer von der Initiative „Datenschutz geht zur Schule“ des BvD.

Wie in vielen anderen Themenfeldern mit direktem Bezug zu Kindern wird auch beim Thema Datenschutz in der Regel nur über die Betroffenen geredet und nicht mit ihnen. Dass es auch anders geht, bewies die heutige Veranstaltung in der niedersäch- sischen Landesvertretung in Berlin, an der rund 70 Kinder teilnahmen.

Mit Unterstützung von DsiN und BvD hatten sich zwei Berliner Grundschulklassen auf die Konferenzthemen vorbereitet und ihre Ergebnisse jeweils in einem Forum zur Diskussion mit Experten und den Teilnehmern gestellt. Auch in den zwei Expertenrunden waren Schüler vertreten, die im vergangenen Jahr mit dem Schülerpreis myDigitalWorld ausgezeichnet wurden. „Kinder haben schon heute

Anspruch auf einen Schulunterricht, der Digitalkompetenz im Fachunterricht vermittelt. Es geht um Bildung und Begeisterung für einen verantwortlichen Umgang mit digitalen

Medien. Gerade für Lehrer und Schulen sind praxiserprobte Konzepte wie DigiBitS - Digitale Bildung trifft Schule - daher das A und O“, erklärte Michael Littger (DsiN) im Eröffnungspanel zur Konferenz.

Moderiert von dem erfahrenen Fernsehmoderator Ralph Caspers stand der Dialog zwischen den Kindern und den eingeladenen Experten im Mittelpunkt der Konferenz. Prof. Dr. Friederike Siller von der TH Köln stellte fest: „Kinder haben das Recht, mit Hilfe der digitalen Medien zu kommunizieren, sich zu informieren und ihre Meinung zu sagen. Wer Kinder dabei unterstützen will, muss die Lebenswirklichkeit von Kindern begreifen und von dort ausgehen. Der Datenschutz kann ein starker Partner an der Seite der Kinder zur Gewährleistung ihrer Rechte sein. Daher freue ich mich, wie auch Kinder und Jugendliche in der Diskussion ihre Vorstellungen, Wünsche und Sorgen klar und selbstbewusst formuliert haben und der Dialog nicht an ihnen vorbei, sondern mit ihnen geführt wurde.“

Auch wenn mit dieser Konferenz nur ein kurzes Schlaglicht auf die Problematik geworfen werden konnte, hat die BfDI aus den Erkenntnissen einige Empfehlungen formuliert, um den Datenschutz für Kinder im Umgang mit digitalen Medien zu verbessern. Die Empfehlungen finden Sie auch in Report in diesem Heft (S. 584).

Die Bundesbeauftragte für den Datenschutz und die Informationsfreiheit (BfDI), Andrea Voßhoff 